OPEN ACCESS

Nano Biomed Eng

ISSN 2150-5578 http://nanobe.org

\title{
Doxorubicin Loaded Polyurethanes Nanoparticles
}

Alberto Dessy†, Anna Maria Piras $\dagger$, Michele Alderighi, Stefania Sandreschi, Federica Chiellini*

Laboratory of Bioactive Polymeric Materials for Biomedical and Environmental Applications (BIOlab), Department of Chemistry \& Industrial Chemistry, University of Pisa, Via Vecchia Livornese 1291, 56122 San Piero a Grado, Pisa (Italy)

* Corresponding author: federica @ dcci.unipi.it(Federica Chiellini)

Tel: +39-050-2210305/2/3. Fax: +39-050-2210332.

$\uparrow$ Alberto Dessy and Anna Maria Piras Contributed Equally to This Paper

\begin{abstract}
A poly(ether-ester-urethane) block copolymer based on poly( $\varepsilon$-caprolactone) and polyethylene glycol (PEGCL) was employed for the production of Doxorubicin loaded polymeric nanoparticles to be used as a drug delivery system. Nanoparticles were obtained by means of the nanoprecipitation/ solvent evaporation technique, possessing a spherical shape and an average diameter of about $80 \mathrm{~nm}$. The optimization of the formulation process led to high formulation yield and appreciable drug content. One of the main findings arising from the present study is represented by the protective activity played by nanoparticles in terms of preventing doxorubicin degradation in physiological conditions; moreover the observed time-delayed release of the drug was associated to PEGCL chemical structure.
\end{abstract}

Keywords: Drug Delivery Systems, Nanoparticles, Cancer, Doxorubicin, Polyurethanes

Citation: Alberto Dessy, Anna Maria Piras, et al. Doxorubicin Loaded Polyurethanes Nanoparticles. Nano Biomed. Eng. 2012, 4(2), 83-88. DOI: $10.5101 /$ nbe.v4i2.p83-88.

\section{Introduction}

Cancer represents one of the major cause of mortality worldwide, with a predicted number of deaths in the European Union of more than 1 million for 2011 [1]; the discovery of cytotoxic drugs represented a revolutionary tool for the treatment of different types of cancer, improving survival rates and quality of life of patients. However, the use of cytotoxic drugs is considerably restricted by frequently associated toxic side effects which are generated by the lack of efficient selectivity for malignant cell [2]. Moreover the low water solubility, rapid phagocytic and renal clearance represent major barriers that limit the use of many hydrophobic antitumour agents [3]. The employment of polymeric nanoparticles as drug delivery systems for the administration of chemiotherapic drugs was demonstrated to improve the anticancer activity and the stability of the loaded drug $[4,5]$. In particular, the entrapment of the drug into the carrier until it is released is desired and can improve the pharmacological properties of traditional chemotherapeutics by altering its pharmacokinetics and biodistribution. Moreover thanks to their small dimensions, polymeric nanoparticles possess the ability to pass through small capillary vessels being able to reach peripheral body district [6]. The feasibility of modifying nanoparticles surface both with hydrophilic protective layer and selective targeting molecules is also important for escaping body's natural defence as well as directing drug loaded nanoparticles to the tumour site [7]. As widely reported by the literature, polymeric nanoparticles can accumulate in solid tumours once they have reached the target site; cancer cells internalize subsequently nanoparticles via the endocytosis route enhancing the specific therapeutic activity of cytotoxic drugs against tumours $[8,9]$.

Many polymeric matrices have been employed for the production of polymeric nanoparticles as drug delivery system; among these, polyurethanes have been largely used for the production of biomedical applications for over 30 years thanks to their non-immunogenic and nontoxic properties [10]. In particular block copolymers based on Poly( $\varepsilon$-caprolactone) (PCL)/ Polyethylene glycol(PEG) poly(ether-ester-urethane)s have drawn increasing research interest for the production of 
drug delivery systems thanks to their biodegradable, biocompatible and amphiphilic properties [11-13].

Doxorubicin, a potent anticancer drug, is widely clinically used in the treatment of various solid tumors of lung and breast as well as leukemia, and lymphomas [14]; its clinical application is, however, limited by the severe side effects, which include specific cytotoxicity and dosedependent congestive heart failure [15].

In the present work the feasibility of obtaining polymeric nanoparticles loaded with doxorubicin, using poly(ether-ester-urethane) PCL/PEG random block copolymer (PEGCL) as polymeric matrix, was investigated.

\section{Experimental Section}

\section{Materials}

1,6-hexamethylenediisocyanate (HMDI), Polyethylene glycol diol (PEG) (MW $2000 \mathrm{Da})$, Poly(E-caprolactone) diol (PCL) (MW2000Da), KCl, $\mathrm{KH}_{2} \mathrm{PO}_{4} \cdot \mathrm{H}_{2} \mathrm{O}, \mathrm{NaCl}$, $\mathrm{Na}_{2} \mathrm{HPO}_{4} \cdot 12 \mathrm{H}_{2} \mathrm{O}$ and $\mathrm{NaOH}$ pellets were supplied by Sigma Aldrich.

PCL/PEG random block copolymer (PEGCL) was synthesized by bulk polymerization by varying the synthesis procedure proposed by Cometa et al [16].

HMDI was distilled at reduced pressure $\left(65^{\circ} \mathrm{C}, 0.2\right.$ mbar).

Phosphate buffer saline solution (PBS 10X) was prepared by dissolving $2.7 \times 10^{-2}$ moles of $\mathrm{KCl}, 1.3 \times 10^{-2}$ moles of $\mathrm{KH}_{2} \mathrm{PO}_{4} \cdot \mathrm{H}_{2} \mathrm{O}, 1.4$ moles of $\mathrm{NaCl}$, and $4.3 \times 10^{-2}$ of $\mathrm{Na}_{2} \mathrm{HPO}_{4} \cdot 12 \mathrm{H}_{2} \mathrm{O}$ in 1 litre of distilled water. The $\mathrm{pH}$ was adjusted to 7.4 with $10 \mathrm{~N} \mathrm{NaOH}$ and the resulting solution was sterilized in autoclave $\left(121^{\circ} \mathrm{C}\right.$ for $\left.20 \mathrm{~min}\right)$ before use and storage. Working solutions were obtained by diluting PBS 10X with distilled water at 1:10 v/v (PBS $1 \mathrm{X})$.

Acetone was purchased by Carlo Erba, Italy; Tryethilamine (TEA) was provided by Mallinckrodt Baker. Doxorubicin-HCl was supplied by Discovery Fine Chemicals, UK. Amicon Ultra-15 Centrifugal filter devices (100 kDa MWCO) was provided by Millipore; Float-A-Lyzer ${ }^{\circledR}$ Cellulose Ester membranes $(10 \mathrm{kDa}$ MWCO) were purchased by Spectrum Laboratories Inc.

\section{Methods}

\section{Doxorubicin loaded PEGCL based polymeric nanoparticles preparation}

$5.1 \times 10^{-6}$ moles of Doxorubicin- $\mathrm{HCl}$ were dissolved into a solution containing $1.1 \times 10^{-3}$ moles of TEA and $4 \mathrm{ml}$ of acetone. After drug dissolution, $1.610^{-6}$ moles of PEGCL polymer were added under mild stirring. The solution was then dropped (using a syringe equipped with a $22 \mathrm{G}$ needle) into $10 \mathrm{ml}$ of deionised water, under mild stirring, with a rate of $30 \mathrm{drops} / \mathrm{min}$. Acetone was removed by evaporation under hood at $40^{\circ} \mathrm{C}$ for 4 hours. The obtained Doxorubicin loaded nanoparticles were then centrifuged using an Amicon Ultra-15 Centrifugal filter device (100 kDa MWCO); three consecutive centrifugal washing steps were performed $\left(20^{\prime}, 8000 \mathrm{rpm}, 4^{\circ} \mathrm{C}\right)$. In order to estimate the formulation yield, nanoparticles were lyophilised. The formulation yield was calculated as the ratio between the dry weight of lyophilised nanoparticles and the sum of the starting weight of each component (Equation 1). The formulation yield was estimated as the mean value of three replicates.

FormulationYield $=\frac{\text { Nanoparticles }(g)}{\sum \text { FormulationComponent }(g)} * 100 \quad$ Equation 1

\section{Nanoparticles physical characterization}

Light scattering analysis and Zeta potential measurements were performed to investigate nanoparticles diameter distribution and surface charge properties; all samples were analysed in triplicate at $25^{\circ} \mathrm{C}$, $\mathrm{pH} 7.4$, using water as a dispersant.

Nanoparticles morphology was analysed by means of Atomic Force Microscopy (AFM). The Microscope was equipped with a type-E scanner stage allowing for measures up to $13 \times 13 \mu \mathrm{m}$ in the XY plane and about 3.5 $\mu \mathrm{m}$ in the $\mathrm{Z}$ axis. AFM images were collected using both RTESP and Olympus Biolever probes. Tapping - mode atomic force microscopy was used to study the structure of nanoparticles deposited onto freshly cleaved HOPG substrate.

\section{Doxorubicin content estimation}

In order to estimate nanoparticles doxorubicin content, dry nanoparticles samples were weighted and completely dissolved in a known amount of acetone/TEA solutions. The drug absorbance value was then measured at $485 \mathrm{~nm}$. Doxorubicin content was estimated by using a calibration curve obtained by plotting the absorbance values at 485 $\mathrm{nm}$ of doxorubicin standard solutions $\left(0.15-25 \mu \mathrm{g} \mathrm{ml}^{-1}\right.$.) vs. their concentration in $\mu \mathrm{g} / \mathrm{ml}\left(\mathrm{R}^{2} 0,999\right)$. All samples were assayed in triplicate. Nanoparticles doxorubicin content was expressed in terms of Encapsulation Efficiency (E.E.\%; Equation 2) and Loading (Ldg.\%; Equation 3).

$$
\begin{aligned}
& \text { E.E.\% }=\frac{\text { DoxorubicinLoaded }(g)}{\text { TotalDoxorubicin }(g)} * 100 \quad \text { Equation } 2 \\
& L d g \%=\frac{\text { DoxorubicinLoaded }(g)}{\text { Nanoparticles }(g)} * 100 \quad \text { Equation } 3
\end{aligned}
$$

\section{Doxorubicin degradation and release kinetic}

The degradation kinetic of Doxorubicin in nanoparticles was assessed by means of UV-Vis spectroscopy by using free Doxorubicin as a reference. Nanoparticles dispersions in $5 \mathrm{ml}$ of PBS $1 \mathrm{X} \mathrm{pH} 7.4$ were obtained from a known amount of nanoparticles (corresponding to a Doxorubicin concentration of $\left.0,07 \times 10^{-2} \mathrm{mM}\right) .0,07 \times$ 
Article

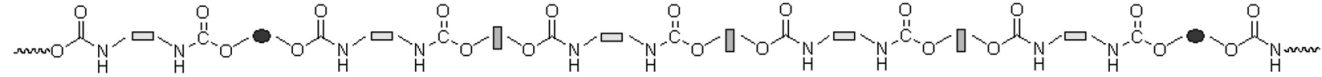

$$
\begin{aligned}
& \text { 口 = Junction Unit (GRMDI) } \\
& \text { [ = Hydrophobic Segment (PCL) } \\
& \text { - = Hydrophilic Segment (PEG) }
\end{aligned}
$$

Fig. 1 PEGCL Random Copolymer Chemical Structure
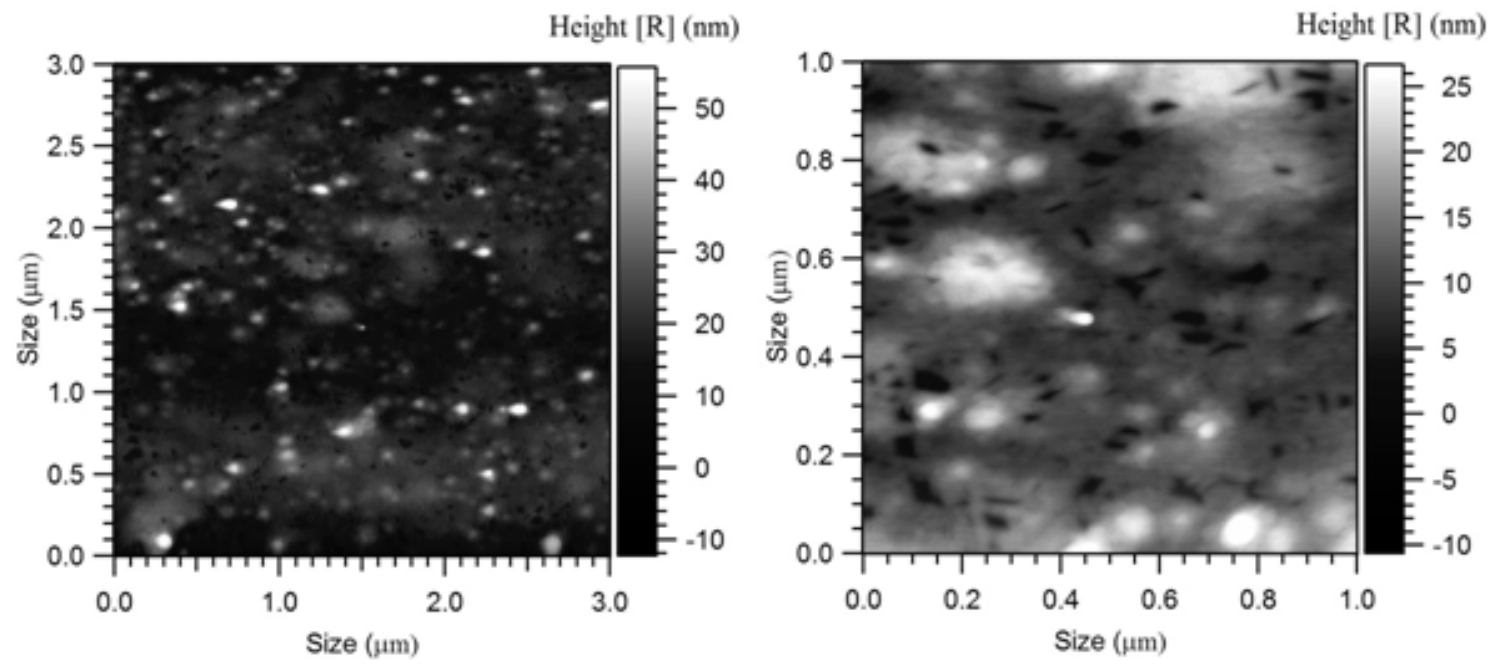

Fig. $23 \times 3 \mu \mathrm{m}$ (left) and 1x1 $\mu \mathrm{m}$ (right) A.F.M. Micrographs of Doxorubicin Loaded Polymeric Nanoparticles

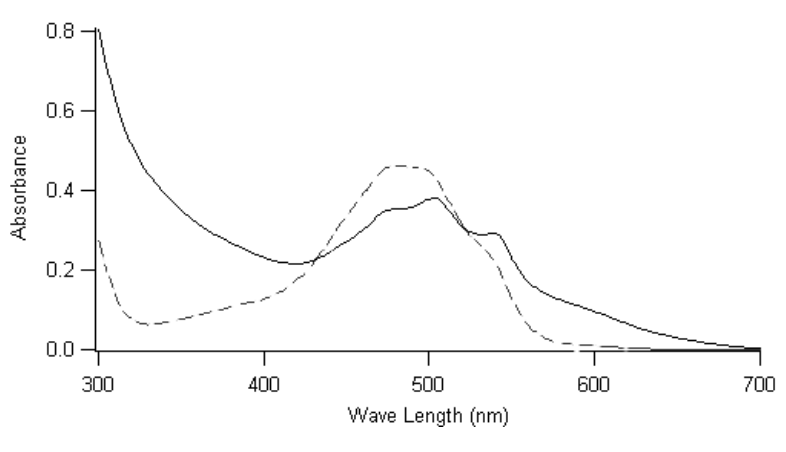

Fig. 3 UV-Vis Absorption Spectrum of Doxorubicin Loaded Nanoparticles (Straight Line) and Free Doxorubicin (Dotted Line).

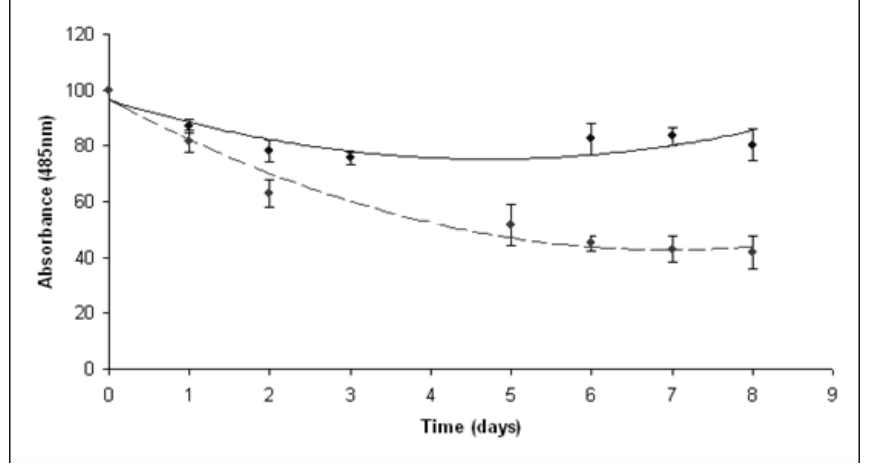

Fig. 4 Doxorubicin Degradation Profile. Free Doxorubicin (Dotted Line), Doxorubicin Loaded Nanoparticles (Straight Line)

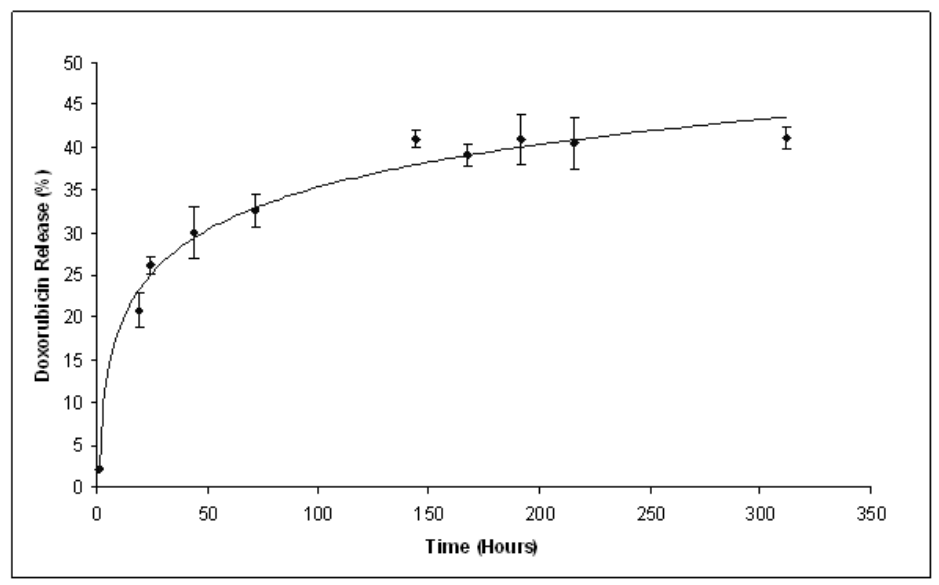

Fig. 5 Doxorubicin Loaded Nanoparticles Release Profile 
Table 1. PEGCL Nanoparticles Loaded with Different Concentration of Doxorubicin

\begin{tabular}{|l|l|l|l|}
\hline Doxorubicin(moles) & E. E. $(\% \pm$ s. d. $)$ & Ldg $(\% \pm$ s. d. $)$ & Size (nm - P. I. ) \\
\hline $1.27 \times 10^{-6}$ & $23 \pm 0.7$ & $0.3 \pm 0.03$ & $75-0.17$ \\
\hline $1.7 \times 10^{-6}$ & $20 \pm 1$ & $0.5 \pm 0.07$ & $73-0.161$ \\
\hline $2.55 \times 10^{-6}$ & $25 \pm 1.3$ & $0.8 \pm 0.05$ & $80-0.132$ \\
\hline $5.1 \times 10^{-6}$ & $26 \pm 0.8$ & $2 \pm 0.2$ & $82-0.154$ \\
\hline
\end{tabular}

$10^{-2} \mathrm{mM}$ Doxorubicin in PBS $1 \mathrm{X} \mathrm{pH} 7.4$ was tested as a reference.

Both the solutions and dispersions were kept in the dark under mild stirring at $37^{\circ} \mathrm{C}$. At regular time intervals UVVis absorbance spectra were performed.

Doxorubicin release kinetic was assessed on doxorubicin loaded nanoparticles dispersions. Donors dialysis volumes of $5 \mathrm{ml}$ containing PBS 1X Doxorubicin loaded nanoparticles suspension and PBS $1 X$ Doxorubicin solution were used. The suspensions were obtained by dispersing a known amount of nanoparticles (corresponding to a Doxorubicin concentration of 0,07 $\mathrm{x} 10^{-2} \mathrm{mM}$ ) in $5 \mathrm{ml}$ of PBS $1 \mathrm{X} \mathrm{pH} \mathrm{7.4.} \mathrm{Dialysis} \mathrm{was}$ achieved through a Float-A-Lyzer ${ }^{\circledR}$ Cellulose Ester membrane $(10 \mathrm{kDa} \mathrm{MWCO})$ into a $50 \mathrm{ml}$ acceptor volume of PBS $1 \mathrm{X} \mathrm{pH} \mathrm{7.4,} \mathrm{stirred} \mathrm{and} \mathrm{thermostated} \mathrm{at}$ $37^{\circ} \mathrm{C}$ in the dark. At regular time intervals, Doxorubicin quantification was performed by UV-Vis spectroscopy of the solutions in the acceptor compartment. All samples were analysed in triplicate.

\section{Instrumentation}

NMR spectra were recorded on Varian Gemini 200 spectrometer using a Sparc 4 console and VNMR 6.1B software. Spectra were processed by using MacFID 1D 5.3 (Tecmag Inc.) software. NMR spectra were recorded on $5-10 \%(\mathrm{w} / \mathrm{v})$ solutions, in deuterated $(\mathrm{CDCl} 3)$ solvent, at $25^{\circ} \mathrm{C}$, with tetramethylsilane (TMS) as internal standard. ${ }^{1} \mathrm{H}-\mathrm{NMR}$ spectra were recorded at $200 \mathrm{MHz}$, using the following spectral conditions: $3 \mathrm{kHz}$ spectral width, $30^{\circ}$ impulse, $2 \mathrm{~s}$ acquisition time, and 32 transients.

Beckman Coulter Delsa ${ }^{\mathrm{TM}}$ Nano C Particle Analyzer instrument was used to investigate nanoparticles dimensional and surface charge (Zeta Potential) properties.

UV-Vis Spectra analysis were performed by means of JascoV-530 spectrophotometer.

Purified Nanoparticles were lyophilised using a 5 Pascal Lio 5P Lyophilizator.

AFM analysis were carried out using a multimode microscope working with a Nanoscope IV controller,
Veeco now Bruker Instr. Santa Barbara Ca.

\section{Results and Discussion}

In the present work nanoparticles loaded with doxorubicin were prepared by means of the nanoprecipitation/solvent evaporation technique. The nanoprecipitation technique, whose use has been widely reported for many polymers including poly(lactides), offers numerous advantages basically related to its easiness in procedure and to its high reproducibility [17].

The random block copolymer PEGCL $(\mathrm{Mn}=18 \mathrm{Kda}$, $\mathrm{PI}=1.8)$, possessing a PCL/PEG molar ratio of 2.4, was synthesized in house (Fig. 1).

Nanoparticles diameter distribution was investigated by means of light scattering analysis which revealed a monomodal distribution with an average diameter of 82 nm (P.I. 0.154).

Nanoparticles morphology was detected by means of Atomic Force Microscopy (A.F.M.) analysis; as shown in Fig. 2 nanoparticles possess a spherical and homogenous morphology which assign them suitable features to be used as injectable system.

The optimization of the formulation and purification process led to a high formulation yield ( $88 \% \pm 2.5$ ). Since drug content is a crucial issue for a drug delivery system, different drug concentrations were tested in the formulation trials in order to maximize nanoparticles doxorubicin content (Table 1).

The employment of $5.1 \times 10^{-6}$ moles of doxorubicin in the formulation system was found to give the best results in terms of encapsulation efficiency $(26 \% \pm 0.8$.) and loading $(2 \% \pm 0.2)$ without altering nanoparticles spherical morphology and size.

Zeta Potential of Doxorubicin loaded nanoparticles was also investigated to check nanoparticles surface properties. Zeta potential measurements revealed average values of about $+14.28 \pm 1.21 \mathrm{mV}$. These results suggest the presence of Doxorubicin molecules possibly adsorbed onto nanoparticles surface. Previous analysis performed on empty nanoparticles revealed, in fact, a Zeta potential average value of about $-9.03 \pm 0.42 \mathrm{mV}$. This negative net 
charge can be attributed to carboxylic moieties exposure arising from the hydrolysis of terminal HMDI units. Since in the range of $\mathrm{pH}$ values included between 6 and 7.5, an equilibrium between the positively-charged and the neutral form of doxorubicin is present [18], the increase in the nanoparticles Zeta potential values when loaded with doxorubicin can be attributed to a partial drug exposure on particles surface.

The effective drug encapsulation inside nanoparticles core can be investigated by UV-Vis spectra analysis. It is known that doxorubicin molecule bears a cromophore group composed of three aromatic anthraquinonic ring, which can be analysed to monitor its interaction with the local chemical microenvironment [19]. In particular the visible absorption spectrum of physically entrapped doxorubicin presents differences if compared to the free doxorubicin one; an evident red-shift of the absorption spectrum is known to characterize micro/nanostructures loaded doxorubicin. This phenomenon is due to a local increasing of doxorubicin concentration which affect the $\pi-\pi$ stacking [20].

The UV-Vis absorption spectrum of doxorubicin loaded PEGCL nanoparticles was investigated. As shown in Figure 3, a marked redshift is clearly evident for doxorubicin loaded nanoparticles absorption spectrum in respect to the one of the free drug. It is thus possible to assume that during nanoparticles formulation process, a fraction of the drug is physically entrapped into nanoparticles structures. This is a key issue when developing drug delivery systems, since it would assure a time controlled delivery of the loaded drug.

Since Doxorubicin present a low stability at $\mathrm{pH}$ values beyond 7 [21, 22], the encapsulation of the drug into nanoparticles structures can prevent its degradation assuring the maintenance of the therapeutic activity.

In order to confirm the proposed theory, the absorbance spectrum of free doxorubicin and doxorubicin loaded nanoparticles was monitored in physiological conditions (PBS, $1 \mathrm{X} \mathrm{pH} 7.4 .37^{\circ} \mathrm{C}$ ) at regular time intervals. As reported in Figure 4 a marked progressive decrease in UV absorbance value at $495 \mathrm{~nm}$ is evident for free doxorubicin, reaching the $40 \%$ after 6 days of incubation; on the other side, only $20 \%$ of $495 \mathrm{~nm}$ absorbance value decay was measured when the drug is loaded into nanoparticles.

These findings attribute to PEGCL based nanoparticles a protective activity against doxorubicin chemical degradation. Since hydroxyl ions are proposed to be involved in Doxorubicin degradation mechanism [21], it is possible to speculate that the drug is effectively loaded inside nanoparticles hydrophobic tasks resulting as a consequence inaccessible for hydroxyl ions. Taking into account that the degradation of Doxorubicin was demonstrated to be fast once injected in the blood stream, with a $10 \%$ degradation after 48 minutes [23], the PEGCL based nanoparticles protective properties can improve doxorubicin activity once injected in-vivo.

To check the release profile of doxorubicin from nanoparticles, a dialysis experiment was performed. As shown in Figure 5, a progressive drug diffusion which reached the $40 \%$ of the loaded drug after 6 days of incubation (150 hours) was observed.

Data reported by the literature, concerning the release of doxorubicin from PCL/PEG/PCL triblock copolymer based nanoparticles, showed a $70 \%$ release of drug after $10 \mathrm{~h}$ of incubation [2]. The random distribution of PEG and PCL moieties all along the PEGCL polymeric backbone seems to be responsible for the time-delayed doxorubicin diffusion observed in the present work. The regular alternation of hydrophilic and hydrophobic moieties present in PCL/PEG diblock copolymers and PCL/PEG/PCL triblock copolymers can in fact create hydrophilic channels which accelerate the drug diffusion from nanoparticles; on the other side the random alternation of PEG and PCL moieties, characterizing PEGCL copolymer, generates extended hydrophobic regions capable of keeping the drug into nanoparticles for longer periods. At the same time PEG rich moieties assure stealth properties and give nanoparticles physical stability once in aqueous environment.

\section{Conclusions}

Poly( $\varepsilon$-caprolactone) / Polyethylene glycol poly(etherester-urethane) (PEGCL) polymer was employed for the production of Doxorubicin loaded nanoparticles. Nanoparticles possessing suitable features to be used as anticancer drug delivery injectable systems in terms of dimension, morphology and drug content were obtained. Doxorubicin chemical stability was demonstrated to be increased when loaded into PEGCL based nanoparticles, improving the drug potential therapeutic activity for the treatment of cancer. The chemical composition of the polymer was hypothesized to be directly responsible for the obtainment of the results shown in the present work. In particular, one of the main advantages arising from the employment of a random block copolymer for the production of drug delivery systems, was noticed to be related to the gradual release of the loaded drug from

\section{Acknowledgements}

The present research was performed within the framework of the European Commission funded project "Nanother" FP7-NMP Theme 2007-4.0-4

\section{References}

1. Malvezzi M, Arfè A, Bertuccio P, Levi F, La Vecchia C, Negri E. European cancer mortality predictions for the year 2011. Ann Oncol. 2011; 22: 947-956. http://dx.doi.org/10.1093/annonc/ mdq774

2. Gou M, Zheng X, Men K, Zhang J, Zheng L, Whang X et al. Poly(E-caprolactone)/Poly(ethylene glycol)/Poly(E-caprolactone) Nanoparticles: Preparation, Characterization, and Application in Doxorubicin Delivery. J Phys Chem. 2009; 113:12928-12933. http://dx.doi.org/10.1021/jp905781g

3. Nasongkla N, Shuai X, Ai H, Weinberg BD, Pink J, Boothman DA et al. cRGD-Functionalized Polymer Micelles for Targeted 
Doxorubicin Delivery. Angew Chem. 2004; 6:6483-6487. http://dx.doi.org/10.1002/ange.200460800

4. Park JH, Lee S, Kim J, Park K. Polymeric nanomedicine for cance therapy. Prog Polym Sci. 2008; 33:113-137.http://dx.doi. org/10.1016/j.progpolymsci.2007.09.003

5. Ganta S, Devalapally H, Shahiwala A, Amiji M. A review of stimuliresponsive nanocarriers for drug and gene delivery. $J$ Controlled Release. 2008; 126: 187-204. http://dx.doi.org/10.1016/ j.jconrel.2007.12.017

6. Brewer E, Coleman J, Lowman A. Emerging technologies of polymeric nanoparticles in cancer drug delivery. J Nanomater. 2011; 2011:1-10. Doi: 10.1155/2011/408675

7. Brigger I, Dubernet C, Couvreur P. Nanoparticles in cancer therapy and diagnosis. Adv Drug Del Rev. 2002;54(5):631-651.http://dx.doi. org/10.1016/S0169-409X(02)00044-3

8. Kwon GS, Okano T. Polymeric micelles as new drug carriers. Adv Drug Del Rev. 1996; 21: 107-116.http://dx.doi.org/10.1016/S0169409X(96)00401-2

9. Maeda H, Wu J, Sawa T, Matsumura Y, Hori K. Tumor vascular permeability and the EPR effect in macromolecular therapeutics: a review. J Controlled Release. 2000; 65: 271-284.http://dx.doi. org/10.1016/S0168-3659(99)00248-5

10. Sun X, Gao H, Wu G, Wang Y, Fan Y, Ma J. Biodegradable and temperature-responsive polyurethanes for adriamycin delivery. Int $J$ Pharm. 2011; 412: 52-58.http://dx.doi.org/10.1016/j.ijpharm.2011. 04.007

11. Moein Moghimi S, Hunter AC, Murray JC. Long-Circulating and Target-Specific Nanoparticles: Theory to Practice. Pharmacol Rev. 2001; 52: 283-318

12. Kim SY, Lee YM, Baik DJ, Kang JS. Toxic characteristics of methoxy poly(ethylene glycol)/poly(epsilon-caprolactone) nanospheres; in vitro and in vivo studies in the normal mice. Biomaterials. 2003;1:55-63.http://dx.doi.org/10.1016/S01429612(02)00248-X

13.Hu Y, Zhang L, Cao Y, Ge H, Jiang X, Yang C. Degradation Behavior of Poly( $\varepsilon$-caprolactone)-b-poly(ethylene glycol)-b-poly( $\varepsilon$ caprolactone) Micelles in Aqueous Solution. Biomacromolecules. 2004;5:1756-1762.http://dx.doi.org/10.1021/bm049845

14. Ying XY, Cui D, Yu L, Du YZ. Solid lipid nanoparticles modified with chitosan oligosaccharides for the controlled release of doxorubicin. Carbohydr Polym. 2011;4:1357-64http://dx.doi. org/10.1016/j.carbpol.2011.01.037

15. Swain SM, Whaley FS, Ewer MS. Congestive heart failure in patients treated with doxorubicin. Cancer. 2003;97:2869-79. http://dx.doi.org/10.1002/cncr.11407
16. Cometa S, Bartolozzi I, Corti A, Chiellini F, De Giglio E, Chiellini E. Hydrolytic and microbial degradation of multi-block polyurethanes based on poly(e-caprolactone)/poly(ethylene glycol) segments. Polym Degrad Stab. 2010;95:2013-21.http://dx.doi. org/10.1016/j.polymdegradstab.2010.07.007

17.Perevyazko I, Vollrath A, Hornig S, Pavlov GM, Schubert US. Characterization of poly(methyl methacrylate) nanoparticles prepared by nanoprecipitation using analytical ultracentrifugation, dynamic light scattering, and scanning electron microscopy. J Polym Sci, Part A: Polym Chem. 2010;48(18):3924-1.http://dx.doi. org/10.1002/pola.24157

18. Tewes F, Munnier E, Antoon B, Ngaboni Okassa L, CohenJonathan S, Marchais $\mathrm{H}$ et al. Comparative study of doxorubicinloaded poly(lactide-co-glycolide) nanoparticles prepared by single and double emulsion methods. Eur J Pharm Biopharm. 2007;66:488 -92.http://dx.doi.org/10.1016/j.ejpb.2007.02.016

19. Prokopowicz M. Synthesis and in vitro characterization of freezedried doxorubicin-loaded silica xerogels. J Microencapsul. 2010;53:525-33.

20.Missirlis D, Kawamura R, Tirelli N, JA. Hubbell. Doxorubicin encapsulation and diffusional release from stable, polymeric, hydrogel nanoparticles. Eur J Pharm Sci. 2006;29(2):120-9. http://dx.doi.org/10.1016/j.ejps.2006.06.003

21. Yokoyama M, Miyauchi M, Yamada N, Okano T, Sakurai Y, Kataoka K et al. Characterization and Anticancer Activity of the Micelle-forming Polymeric Anticancer Drug Adriamycin-conjugated Poly(ethylene glycol)-Poly(aspartic acid) Block Copolymer. Cancer Res. 1990;50(6):1693-700.

22.Munnier E, Cohen-Jonathan S, Linassier C, Douziech-Eyrolles L, Marchais H, Soucé M et al. Novel method of doxorubicinSPION reversible association for magnetic drug targeting. Int $J$ Pharm. 2008;363(1-2):170-6.http://dx.doi.org/10.1016/ j.ijpharm.2008.07.006

23. Laubrock N, Hempel G, Schulze-Westhoff P, Würthwein G, Flege S, Boos J. The stability of doxorubicin and ldarubicin in plasma and whole blood. Chromatographia. 2000;52(1):9-13. http://dx.doi. org/10.1007/BF02490784

Copyright:(c) 2012 Alberto Dessy, Anna Maria Piras, et al. This is an open-access article distributed under the terms of the Creative Commons Attribution License, which permits unrestricted use, distribution, and reproduction in any medium, provided the original author and source are credited. 